D. Schuler, C. Naumann, M. Braun-Unkhoff, F. Zabel, U. Riedel, A Single Pulse Shock Tube Study on the Pyrolysis of 2,5-Dimethylfuran, Zeitschrift für Physikalische Chemie 229 (4), 529-548, 2015

The original publication is available at http://www.degruyter.com/

doi $\underline{\text { 10.1515/zpch-2014-0628 }}$ 
Dominik F. Schuler*, Clemens Naumann, Marina Braun-Unkhoff, Uwe Riedel, and Friedhelm Zabel

\title{
A Single Pulse Shock Tube Study on the Pyrolysis of 2,5-Dimethylfuran
}

\begin{abstract}
The pyrolysis of 2,5-dimethylfuran has been studied in a single pulse shock tube equipped with fast probing device at temperatures between $1175 \mathrm{~K}$ and $1450 \mathrm{~K}$ and pressures of $8.0 \pm 0.5$ bar. The initial concentration of 2,5-dimethylfuran diluted in argon (500 ppm) was much lower than in previous studies reported in the literature. Sixteen different product species were quantified by gas chromatography. The product distribution pattern was compared with the prediction of two comprehensive chemical kinetic reaction mechanisms taken from the literature. In general, the predictions of the mechanisms fit the results of the experiments; however, the comparison reveals some differences between the two mechanisms as well as between simulations and experiments.
\end{abstract}

Keywords: Single Pulse Shock Tube, Chemical Kinetics, 2,5-Dimethylfuran, Pyrolysis.

DOI 10.1515/zpch-2014-0628

Received September 28, 2014; accepted November 4, 2014

Dedicated to Professor Henning Bockhorn on the occasion of his $70^{\text {th }}$ birthday

\section{Introduction}

2,5-Dimethylfuran (2,5-DMF), a possible second generation biofuel, has got some attention lately. It can be obtained from sugar, namely fructose, via two catalytic

*Corresponding author: Dominik F. Schuler, Institut für Verbrennungstechnik, Deutsches Zentrum für Luft- und Raumfahrt e.V. (DLR), Pfaffenwaldring 38-40, 70569 Stuttgart, Germany, e-mail: Dominik.Schuler@dlr.de

Clemens Naumann, Marina Braun-Unkhoff, Uwe Riedel: Institut für Verbrennungstechnik, Deutsches Zentrum für Luft- und Raumfahrt e.V. (DLR), Pfaffenwaldring 38-40, 70569 Stuttgart, Germany

Friedhelm Zabel: Institut für Physikalische Chemie, Universität Stuttgart, Pfaffenwaldring 55 , 70569 Stuttgart, Germany 
hydrolysis steps [1]. The properties of 2,5-DMF are superior to ethanol, the only biofuel used in transport in high quantities nowadays, besides bio-diesel derived from vegetable oils or animal fats. Among those qualities are a higher energy density, a higher research octane number (RON), and a virtual insolubility in water [2].

Several studies of 2,5-DMF pyrolysis and combustion, both theoretical and experimental, have been undertaken, especially in the last years (e.g. Friese et al. [3]). Sirjean et al. [4] gave a comprehensive overview over the results published earlier by different groups.

Product distribution patterns from single pulse shock tubes are valuable data for the validation of reaction mechanisms. Applying the single pulse shock tube technique, the pyrolysis of 2,5-DMF was studied by Lifshitz et al. [5] and by Somers et al. [6].

In their experiments, Lifshitz et al. covered the temperature range from $1070 \mathrm{~K}$ to $1370 \mathrm{~K}$ at pressures between 2 and 4 bar, with initial concentration of $5000 \mathrm{ppm}$ 2,5-DMF. They identified and quantified a large number of pyrolysis products. For temperature calibration, Lifshitz et al. used the decomposition reaction of 1,1,1-trifluorethane (1,1,1-TFE) to 1,1-difluorethene and hydrogen fluoride.

Quite recently, quantum chemistry calculations by Sirjean et al. [4] of the decomposition reaction of 1,1,1-TFE indicate that the rate coefficient used by Lifshitz et al. was off by a factor of 3.8 at $1200 \mathrm{~K}$ and 2.5 bar. Hence, they proposed to correct the temperature range given for the decomposition of 2,5-DMF to $1125-1450 \mathrm{~K}$, instead of $1070-1370 \mathrm{~K}$. Somers et al. [6] covered the temperature range from $1200 \mathrm{~K}$ to $1325 \mathrm{~K}$, and verified the temperature correction of Sirjean et al. They used an initial concentration of $30000 \mathrm{ppm}$ 2,5-DMF. The pressure behind the reflected shock was between 2 and $2.5 \mathrm{~atm}$.

In the present study, also performed in a single pulse shock tube, the experimental conditions differ slightly from those of Lifshitz et al. and Somers et al., with respect to initial concentration, temperature, and pressure. The initial 2,5-DMF concentration is much lower at only $500 \mathrm{ppm}$, the temperature ranges from $1175 \mathrm{~K}$ to $1450 \mathrm{~K}$, and the pressure behind the reflected shock is around a factor of four higher i.e. $8.0 \pm 0.5$ bar.

Sirjean et al. published a chemical reaction mechanism describing the pyrolysis and oxidation kinetics of 2,5-DMF featuring 294 species and 1459 reactions [4], below referred to as Nancy 2013. The model consists of three parts: (i) a base mechanism originally designed for describing the combustion of toluene; (ii) the primary mechanism dealing with the consumption of 2,5-DMF; and (iii) a secondary mechanism for products formed in the primary mechanism and not included in the base mechanism. The initial steps of the 2,5-DMF pyrolysis include carboncarbon and carbon-hydrogen bond fissions in the methyl groups as well as ring 
opening and formation of carbonic species by intramolecular hydrogen atom or methyl group transfer. The Nancy 2013 model is based on quantum chemical calculations and was validated by comparison to ignition delay time measurements and to the temperature corrected single pulse measurements of Lifshitz et al. [5].

The second reaction mechanism of 2,5-DMF combustion considered in the present work was published by Somers et al. [6], below referred to as Galway 2013. The Galway 2013 mechanism contains 545 species and 2768 reactions. The mechanism was validated by comparison to measurements of (i) ignition delay times determined in a low as well as in a high pressure shock tube; (ii) laminar burning velocities measured in a flat-flame burner; (iii) species profiles obtained by jet-stirred reactor and single pulse shock tube experiments. Note that the more recent changes to the reaction mechanism published in a study on 2-methylfuran [7] have very little impact on the simulation results shown in the current work. Therefore the results are not included within the present work. For more details, refer to [8].

\section{Experimental}

The pyrolysis of 2,5-dimethylfuran was investigated in a single pulse shock tube behind reflected shock waves at temperatures between $1175 \mathrm{~K}$ and $1450 \mathrm{~K}$ and at pressures of $8.0 \pm 0.5$ bar. The results of these experiments are product distribution patterns of the 2,5-DMF pyrolysis - about 16 species up to toluene - obtained by fast probing followed by GC/MS analysis.

\subsection{Single pulse shock tube}

All experiments were performed in the stainless steel single pulse shock tube of DLR Stuttgart's Institute of Combustion Technology. The length of the driven section is $489 \mathrm{~cm}$, the driver section's length is adjustable and was fixed mostly at a length of around $240 \mathrm{~cm}$. An intermediate section of $10 \mathrm{~cm}$ length is located between the driver and the driven section. The inner diameter of all three parts is $4.6 \mathrm{~cm}$. The sections are separated by aluminum membranes (thickness $150 \mu \mathrm{m}$ ). Prior to the shock initiation, the intermediate volume was pressurized up to half of the final driver pressure. Helium was used as driver gas. The shock was initiated by releasing the pressure of the intermediate volume resulting in successive bursting of both membranes. This method allows for a better control of the initial temperature and pressure at successive experiments. 


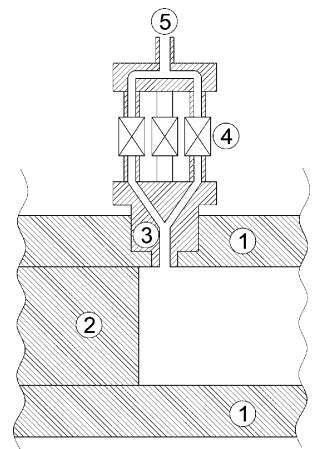

Figure 1: Cross section of end of the shock tube (1) with the endplate (2) and the fast probing device (3) with the fast valves (4) and the outlet (5) to the sample volume.

The whole apparatus can be heated to $473 \mathrm{~K}$ to prevent condensation. For all experiments of the present work, the temperature of the tube was adjusted to $393 \mathrm{~K}$.

As the single pulse shock tube used in the present work has a fast probing device, located at the side wall of the driven section $10 \mathrm{~mm}$ before the end plate, there is no need for a dump tank, as the classical setup requires. The probing device (Figure 1) consists of a $4 \mathrm{~mm}$ bore, splitting up into 4 tubes with an inner diameter of $3 \mathrm{~mm}$ each leading to an electrical driven fast probing valve (Parker Hannifin Corporation type: 009-0209-900). The total dead volume between the tube and valves (bore and tubes) is $1.8 \mathrm{~cm}^{3}$. Behind the valves the flows rejoin ending in a sample volume of $66.7 \mathrm{~cm}^{3}$. The use of four parallel valves becomes necessary to ensure the high mass flow needed due to the short sampling time. The valves were triggered to open behind the reflected shock wave and to close before the re-reflected shock arrives. After the valves received the switching pulse (Figure 2), they take roughly $1 \mathrm{~ms}$ to open, the closing after the pulse wears off $5 \mathrm{~ms}$ later takes however $4 \mathrm{~ms}$. The total sampling time is around $8 \mathrm{~ms}$. The stainless steel sample volume, which is evacuated via a spiro pump (Alcatel type: Drytel 30) prior to use is equipped with a septum and a pressure sensor (Schaevizt Sensors type: P014-0002, range: $0-1$ bar). The sampling device and the probing volume were heated to the same temperature as the shock tube (393 K).

The velocity of the incident shock was measured by eight piezo electric pressure transducers (PCB type: 112A05) along the shock tube. The temperature and pressure behind the reflected shock $\left(T_{5}, p_{5}\right)$ were calculated using the RankineHugoniot equations; for details, see $[9,10]$. 


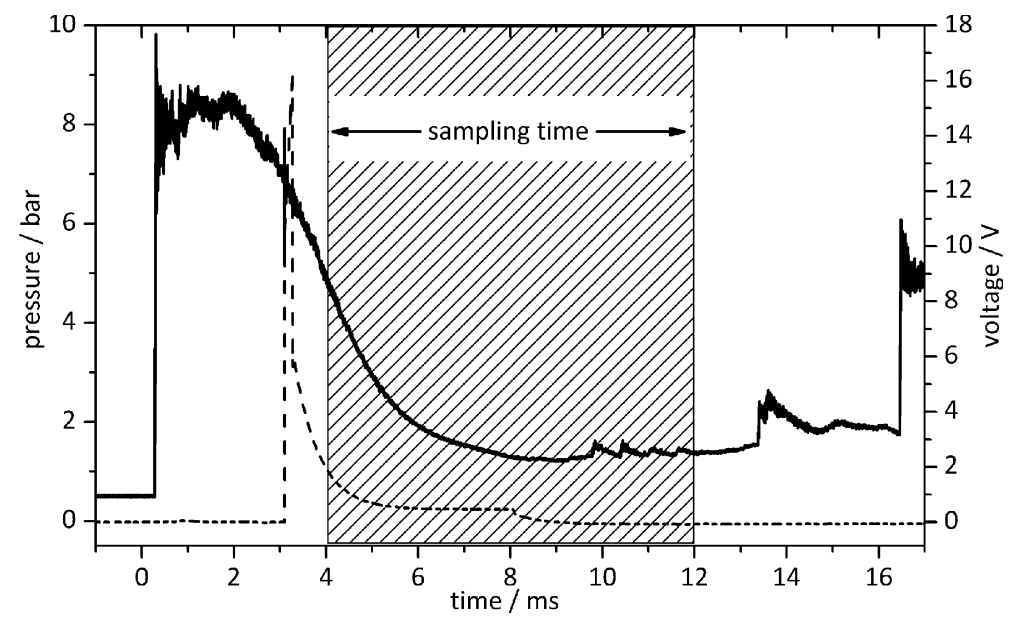

Figure 2: Timing of the probing: pressure in the measurement plane (full line), switching pulse (dashed line), and sampling time (shaded).

\subsection{Test gas mixtures}

The test gas mixtures consist of around 500 ppm 2,5-dimethylfuran and $2000 \mathrm{ppm}$ of xenon diluted in argon (supplied by Linde Gas with a purity of 99.9999\%). Xenon works as an internal standard for the analysis of the sample. The 2,5-dimethylfuran (distributed by VWR with a purity of $99 \%$ ) was distilled prior to use. A mixture of xenon (4950 ppm diluted in Argon) was supplied by Linde Gas. The mixtures were prepared in a stainless steel vessel at $393 \mathrm{~K}$ and allowed to mix for at least three hours. The mixtures' integrity was checked by GC/MS-analysis.

\subsection{Analytic facilities}

The gas samples resulting from the single pulse experiments were analyzed using a gas chromatograph combined with a mass spectrometer as detector (GCMSQP2010 Plus, Shimadzu). Helium (ECD grade, delivered by Linde Gas) was the carrier gas, xenon the internal standard. Two different columns were applied to separate the sample: The Zebron column (ZB-WAXplus) by Phenomenex, and the GS-GASPRO column by J\&W Scientific for $\mathrm{C}_{2}-\mathrm{C}_{8}$ species and $\mathrm{CO}$, respectively. The sample was injected into the GC via gas tight syringes. The syringes were stored at $383 \mathrm{~K}$ prior to use to avoid condensation.

The chemicals used to calibrate the GC/MS were provided by different suppliers: Linde Gas delivered ethane, ethene, ethyne, propene, propyne, allene, 1-butene, 2-butyne, 1,3-butadiene, and but-1-en-3-yne, all of them diluted in ni- 
trogen to a concentration of around $500 \mathrm{ppm}$. Also from Linde Gas, pure carbon monoxide 99.997\% was obtained. 2-Methylfuran 99\% was delivered by Aldrich. 1,3-Cyclopentadiene was synthesized by cracking dicyclopentadiene at $450 \mathrm{~K}$ and separated by distillation from the educt. 1,3-Butadiyne was freshly synthesized from but-2-yne-1,4-diol via 1,4-dibrom-but-2-yne [11, 12].

\subsection{Temperature calibration}

Of course, as in all single pulse shock tubes, the sample obtained from the tube is a mixture both in time and space. The temperature $T_{5}$ is only valid in the center of the tube and not near the walls and only in the instant directly after the shock. For the chronological variance of the temperature we use temperature profiles (see section simulation). The spatial distribution of the temperature cannot be resolved making a temperature calibration necessary. The wanted calibration function $T_{5}^{*}=f\left(T_{5}\right)$ should have the following characteristics:

- $T_{5}^{*}<T_{5}$ as there is no way that the average temperature over space could be higher than in the center,

- $\frac{\partial T_{5}^{*}}{\partial T_{5}}>0$ for $0 \mathrm{~K} \leq 1700 \mathrm{~K}$ as there should be no extreme values in the domain.

The function $T_{5}^{*}=T_{5} \cdot \exp -\left(T_{5} / a\right)^{n}$ with $a \in \mathbb{R}^{+}$and $n \in \mathbb{N}$ was found to have the right characteristics.

The thoroughly investigated decomposition of dinitrogen monoxide $\left(\mathrm{N}_{2} \mathrm{O}\right)$,

$$
\begin{aligned}
& \mathrm{N}_{2} \mathrm{O}+\mathrm{Ar} \leftrightarrow \mathrm{N}_{2}+\mathrm{O}+\mathrm{Ar}, \\
& \mathrm{N}_{2} \mathrm{O}+\mathrm{O} \leftrightarrow \mathrm{N}_{2}+\mathrm{O}_{2},
\end{aligned}
$$

was used as an external chemical thermometer. The rate coefficients for (R1) were taken from Frank et al. [9] and for (R2) from Tsang et al. [13], respectively:

$$
\begin{aligned}
& k_{\mathrm{R} 1}=(4.7 \pm 1.2) 10^{14} \exp [-(248.8 \pm 3.2) / \mathrm{RT}] \mathrm{cm}^{3} \mathrm{~s}^{-1} \mathrm{~mol}^{-1}, \\
& k_{\mathrm{R} 2}=1.02 \cdot 10^{14} \exp (-117 / \mathrm{RT}) \mathrm{cm}^{3} \mathrm{~s}^{-1} \mathrm{~mol}^{-1},
\end{aligned}
$$

where $R$ expressed in units of $\mathrm{kJ} \mathrm{mol}^{-1} \mathrm{~K}^{-1}$.

Comparison of the experimental values with the simulations (see section simulation procedure) of the decomposition of dinitrogen monoxide were used to identify the parameters of a calibration function $a$ and $n$. The final calibration function reads as:

$$
\begin{aligned}
& T_{5}^{*}=T_{5} \cdot \exp \left[-\left[T_{5} /(2746 \pm 19) K\right]^{5}\right] \\
& \text { for } T_{5}^{*} \leq 1700 \mathrm{~K}
\end{aligned}
$$

where $T_{5}^{*}$ is the calibrated temperature behind the reflected shock. 
All species profiles will be plotted as a function of $T_{5}^{*}$ in the present work. Note that $T_{5}^{*}$ represents only the initial value of a temperature profile $T_{5}^{*}=T(t=0)$.

\subsection{Simulation procedure}

For simulations of the experiments with detailed chemical kinetic reaction mechanisms, an adaptation [14] of the SENKIN code of the CHEMKIN II program suite was used [15]. In addition, Chemical Workbench from KINTECH Laboratory was used for reaction path analysis [16].

For each simulation, the measured pressure profile (Figure 3) of the corresponding experiment was used as an input. The calibrated temperature $T_{5}^{*}$ is then taken as an initial value $T(t=0)$ for a temperature profile $T(t)$ derived by adiabatic isentropic compression from the measured pressure profile. The maximal value of the temperature $T_{\max }(t)$ can be up to $4 \%$ higher than the initial value $T(t=0)$, the lowest temperature at the end of the reaction time is usually around $3 \%$ lower than the initial temperature.

Like in the paper of Hidaka et al. [17] a reaction time for the simulation of each experiment was identified. The time between the arrival of the reflected shock at the fast probing device and the intersection of the pressure at this time with the tangent on the steepest slope of the pressure profile (Figure 3) proved to be a good criterion for the reaction time estimate. It is of course possible to extend the simu-

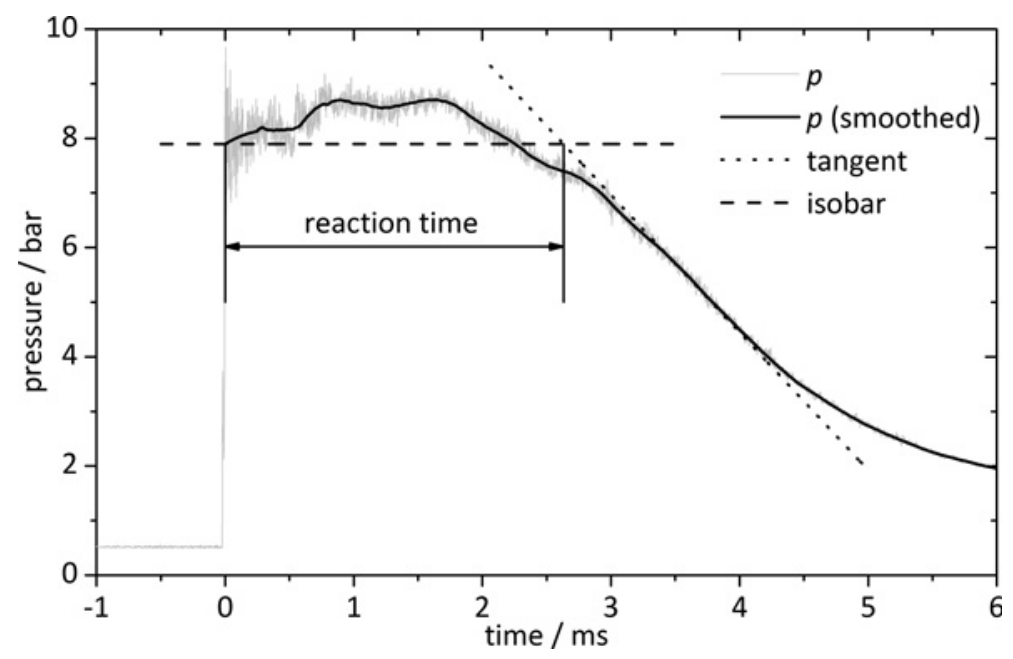

Figure 3: Determination of the reaction time from the measured pressure profile near the endplate. 
lation to longer times and take the complete cooling phase into account; however, the change in concentration of the considered stable species is insignificant.

\section{Results and discussion}

In the present work, the pyrolysis of 2,5-dimethylfuran was studied at combustion relevant temperatures and elevated pressures. The resulting product distribution pattern measured and analyzed by GC/MS was compared to the prediction of two different chemical kinetic reaction models from the literature $[4,6]$ as mentioned earlier.

The results from the gas analysis are normalized to the initial concentration of 2,5-DMF, in order to compare the results of different mixtures at slightly different initial concentrations (full diamonds in the graphs). The dead volume existing between tube and fast valves requires a correction with respect to the exact concentration of the educt and the product species, as described below. At the beginning of an experiment, the dead volume, being open to the shock tube, is filled with test gas. This test gas has no direct contact to the shock and is therefore assumed to be unaltered within the course of an experiment. During probing, this gas is pressed into the probing volume. With all volumes and pressures known, it is possible to calculate and correct for this effect (open diamonds in the graphs).

The dead volume correction effectively decreases the residual 2,5-DMF concentration, thus increasing the product concentrations as the sample is diluted by the gas in the dead volume containing unreacted 2,5-DMF. The assumption made in this correction (the gas in the dead volume does not react at all) may lead to an overestimation of the effect; thus, both the corrected (open diamonds) and the unaltered (full diamonds) values are shown in the graphs as upper and lower limits. The overestimation can be seen in Figure 4 where the dead volume correction leads to negative values.

The measurement uncertainties of the present work were estimated for all experiments. Results of this analysis are included in the figures as error bars, as shown in Figures 3-16 and 18-21 for three temperatures. The error bars given in the ordinate (concentration) include uncertainties in the initial concentrations of 2,5-DMF and xenon, in the calibration of the analytical system as well as uncertainties in the evaluation of the spectra of the gas sample. The error bars given in the abscissa (temperature) include the uncertainty in the measurement of the shock velocity. 


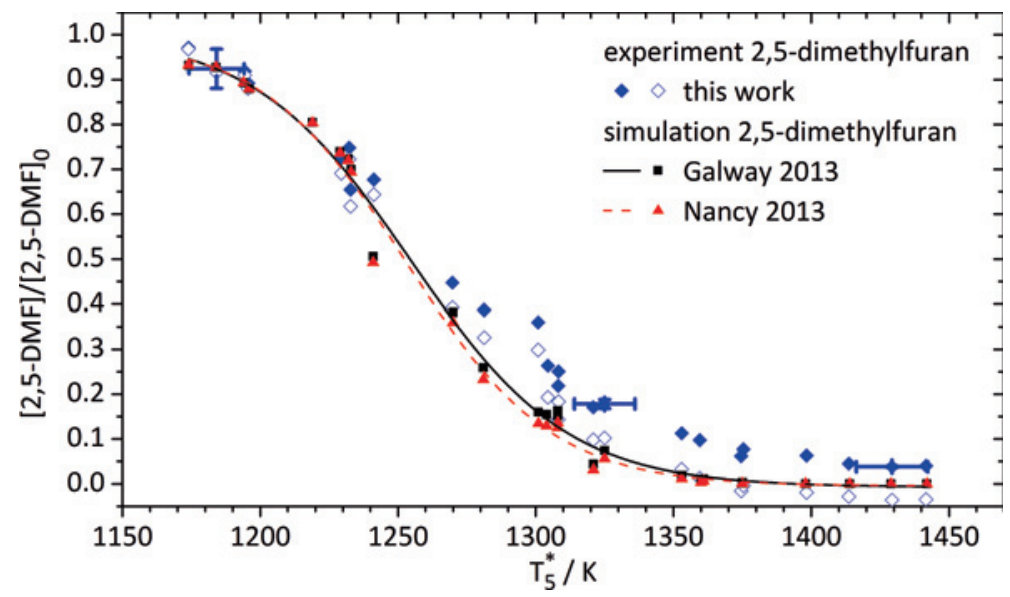

Figure 4: Shock tube decomposition profile for 500 ppm 2,5-DMF in argon: measured (full diamonds) and corrected for dead volume effects (open diamonds). Modeling calculations using chemical kinetic reaction mechanisms from Galway 2013 (squares) [6] and Nancy 2013 (triangles) [4].

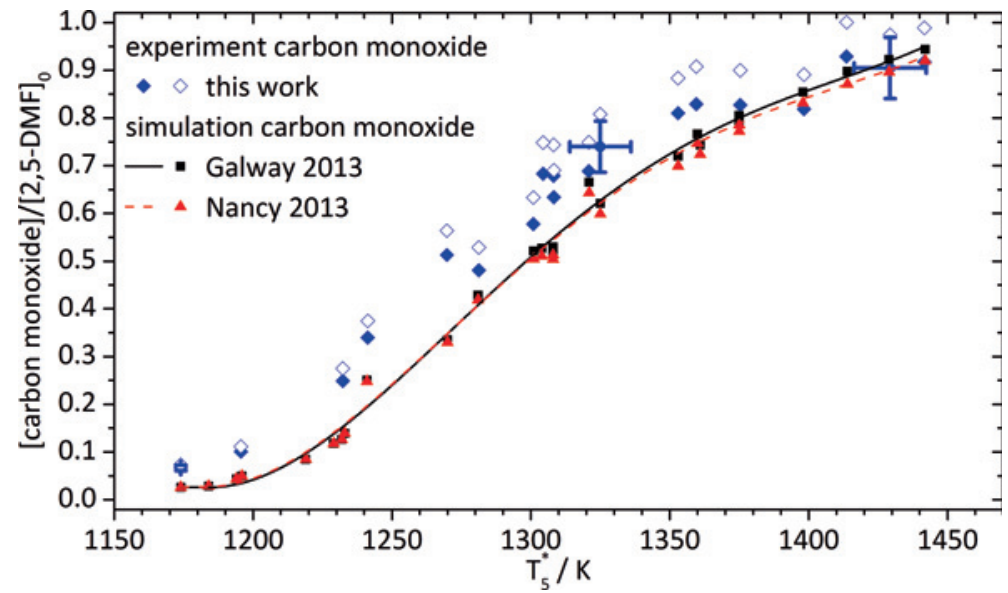

Figure 5: Pyrolysis product carbon monoxide: measured (full diamonds) and corrected for dead volume effects (open diamonds). Simulations: Galway 2013 (squares) [6] and Nancy 2013 (triangles) [4].

For each experiment, the result of the simulations of the two considered reaction mechanisms are denoted in the plots by symbols. The lines in the graphs are only guide lines derived from best fits to the simulation results. 


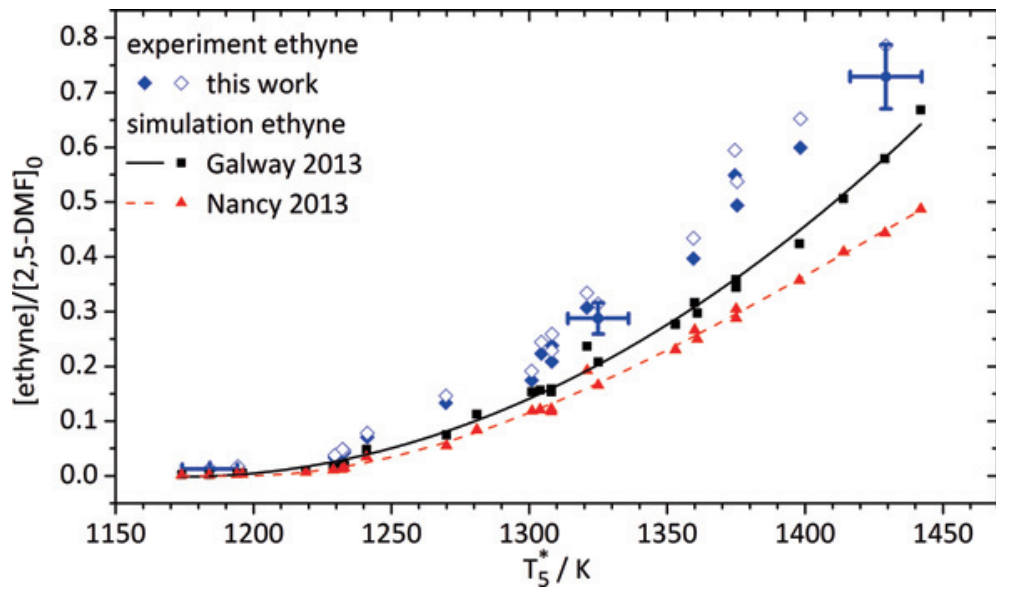

Figure 6: Pyrolysis product ethyne: measured (full diamonds) and corrected for dead volume effects (open diamonds). Simulations: Galway 2013 (squares) [6] and Nancy 2013 (triangles) [4].

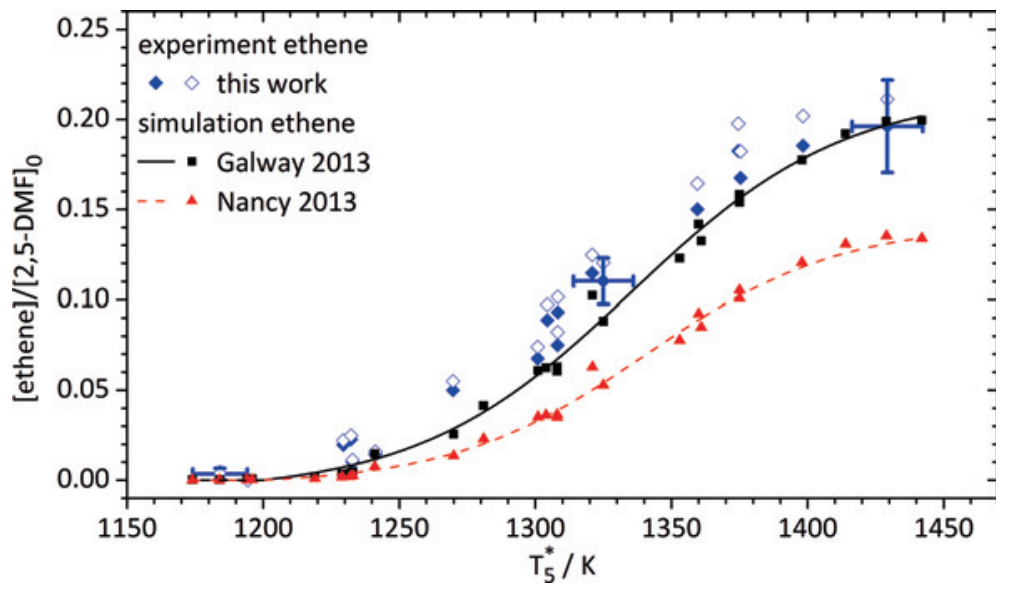

Figure 7: Pyrolysis product ethene: measured (full diamonds) and corrected for dead volume effects (open diamonds). Simulations: Galway 2013 (squares) [6] and Nancy 2013 (triangles) [4].

Simulations of the decomposition of 2,5-DMF (Figure 4) with both mechanisms used, Galway 2013 and Nancy 2013, show good agreement with the experimental values. In both mechanisms, the main degradation pathways of 2,5-DMF under the current conditions are: (i) the hydrogen abstraction from the methyl group to form 5-methyl-2-furanyl-methyl radical, (ii) the ring opening leading to 3,4-hexadiene-2-one, (iii) the addition of a hydrogen atom at the alpha position followed by either the scission of the methyl group (ipso substitution) or (iv) the 


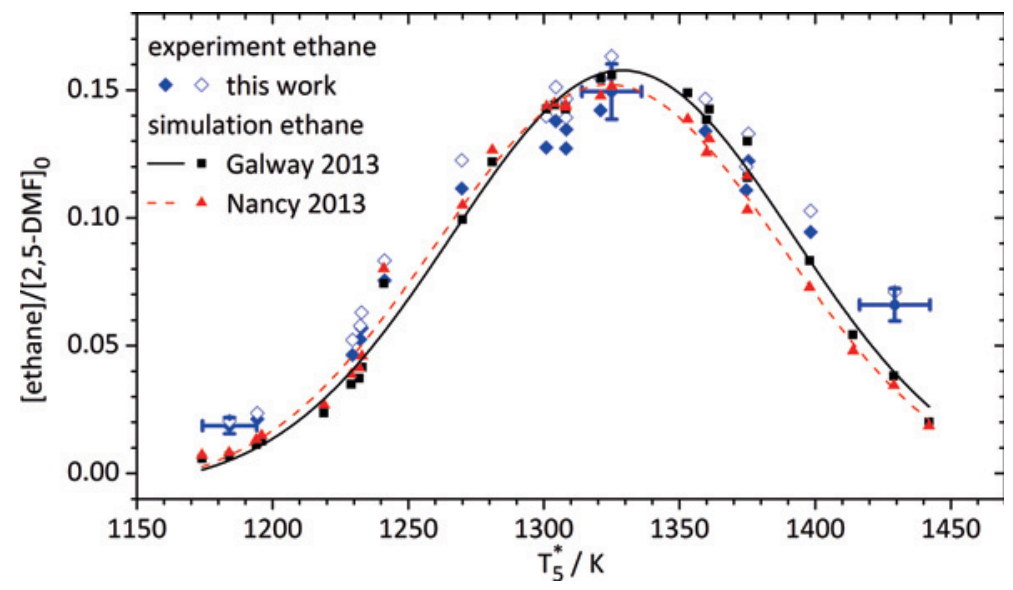

Figure 8: Pyrolysis product ethane: measured (full diamonds) and corrected for dead volume effects (open diamonds). Simulations: Galway 2013 (squares) [6] and Nancy 2013 (triangles) [4].

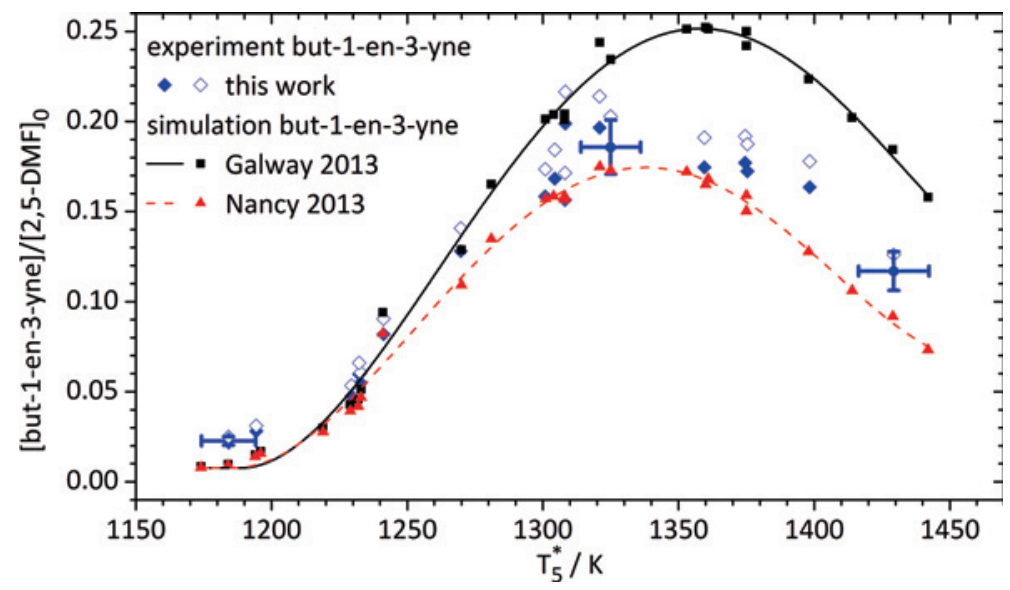

Figure 9: Pyrolysis product but-1-en-3-yne: measured (full diamonds) and corrected for dead volume effects (open diamonds). Simulations: Galway 2013 (squares) [6] and Nancy 2013 (triangles) [4].

decomposition of the intermediate radical to form 1,3-butadiene and an acetyl radical. For reaction schemes, see [4, 6].

Carbon monoxide is the main oxygen containing product species in this study. The formation of carbon monoxide (Figure 5) is underestimated slightly by both mechanisms, over the whole temperature range. The simulations are in very good agreement with each other. 


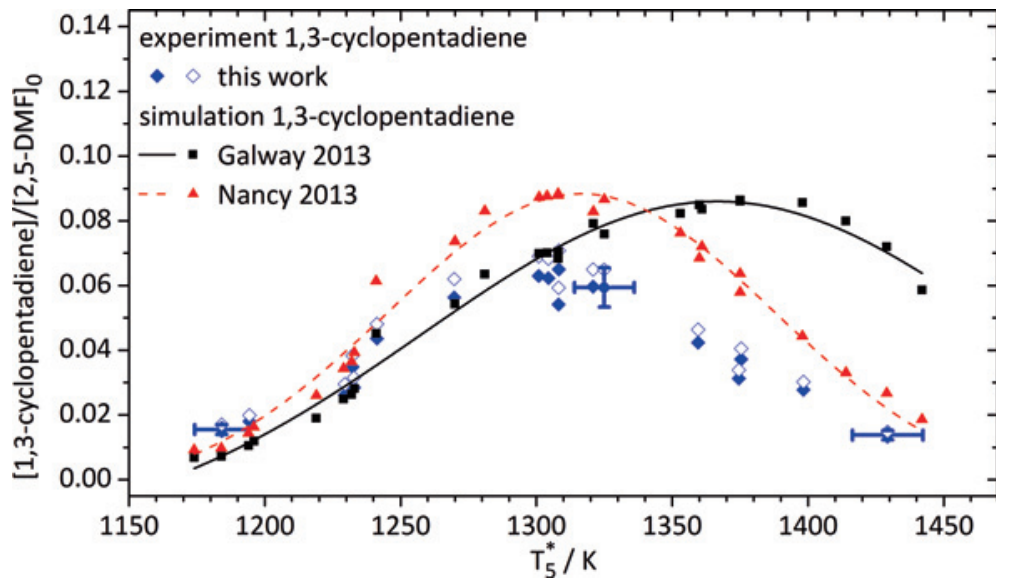

Figure 10: Pyrolysis product 1,3-cyclopentadiene: measured (full diamonds) and corrected for dead volume effects (open diamonds). Simulations: Galway 2013 (squares) [6] and Nancy 2013 (triangles) [4].

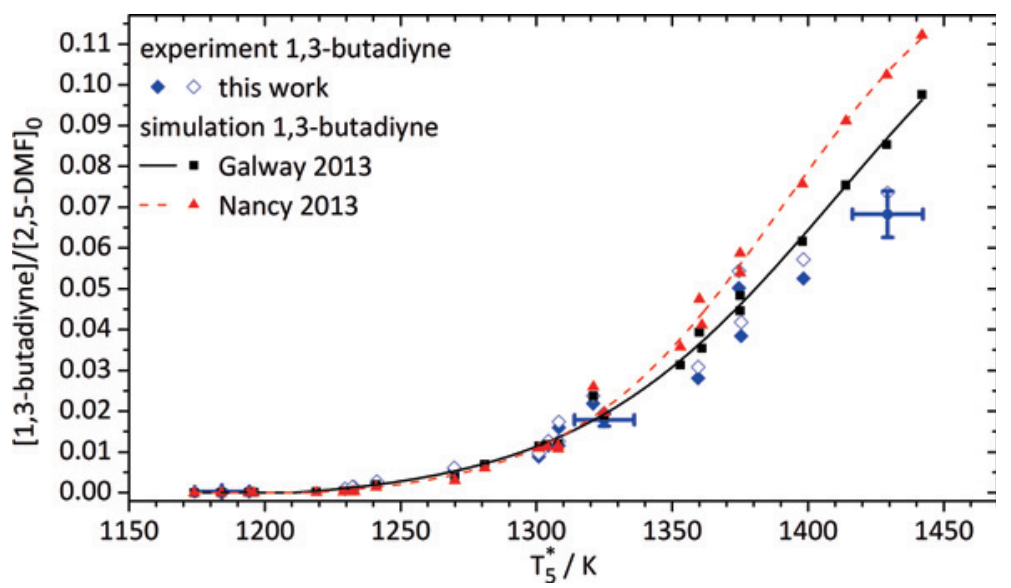

Figure 11: Pyrolysis product 1,3-butadiyne: measured (full diamonds) and corrected for dead volume effects (open diamonds). Simulations: Galway 2013 (squares) [6] and Nancy 2013 (triangles) [4].

The only other oxygen containing species found was 2-methylfuran which is only a minor component under the conditions in this study. The oxygen balance is $93.4 \pm 5.4 \%$.

Ethyne (acetylene) (Figure 6) and ethene (Figure 7) are main products of the 2,5-DMF pyrolysis. Both mechanisms show the same tendency as the experimen- 


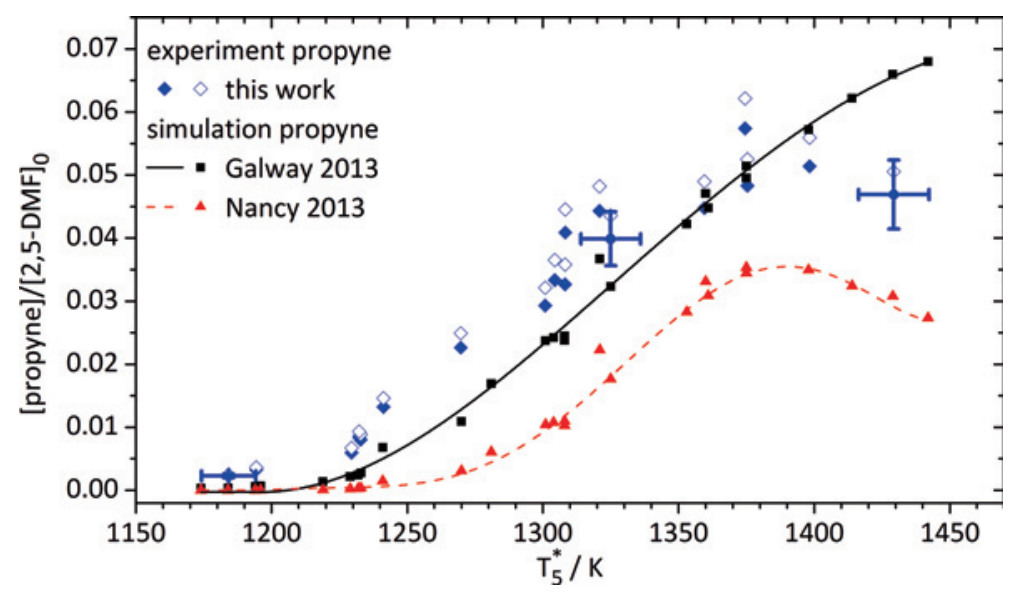

Figure 12: Pyrolysis product propyne: measured (full diamonds) and corrected for dead volume effects (open diamonds). Simulations: Galway 2013 (squares) [6] and Nancy 2013 (triangles) [4].

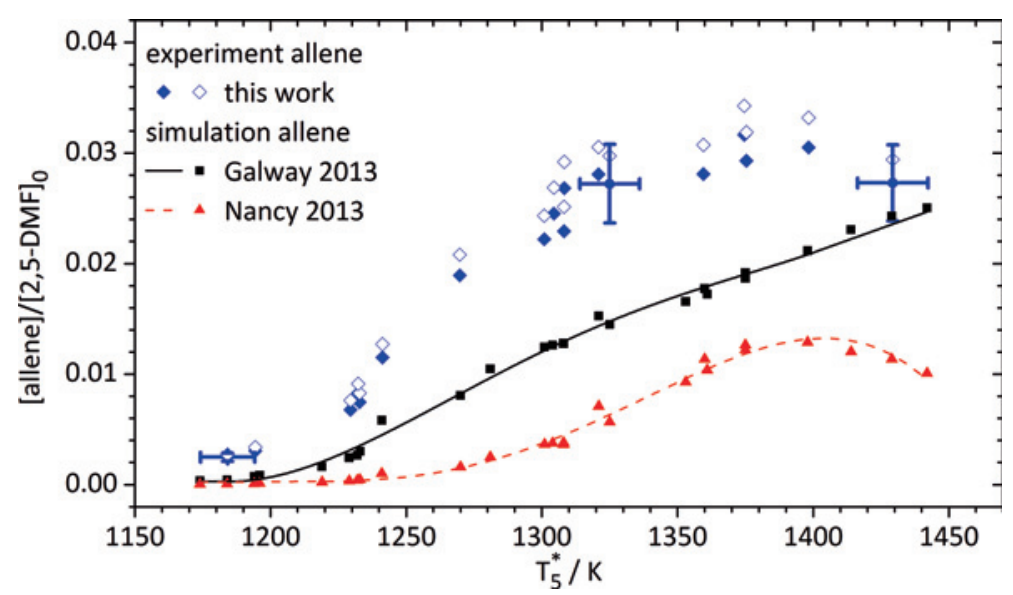

Figure 13: Pyrolysis product allene: measured (full diamonds) and corrected for dead volume effects (open diamonds). Simulations: Galway 2013 (squares) [6] and Nancy 2013 (triangles) [4].

tal values but underestimate the production of ethyne and ethene at higher temperatures. The predictions of Galway 2013 are reproducing the experimental findings with respect to these two species better than the predictions of Nancy 2013.

Moreover, both mechanisms are in very good agreement with the experimental results for ethane (Figure 8) produced during the 2,5-DMF pyrolysis.

For but-1-en-3-yne (Figure 9), the prediction of Nancy 2013 is in good agreement with the experimental values. Galway 2013 reproduces the but-1-en-3-yne 


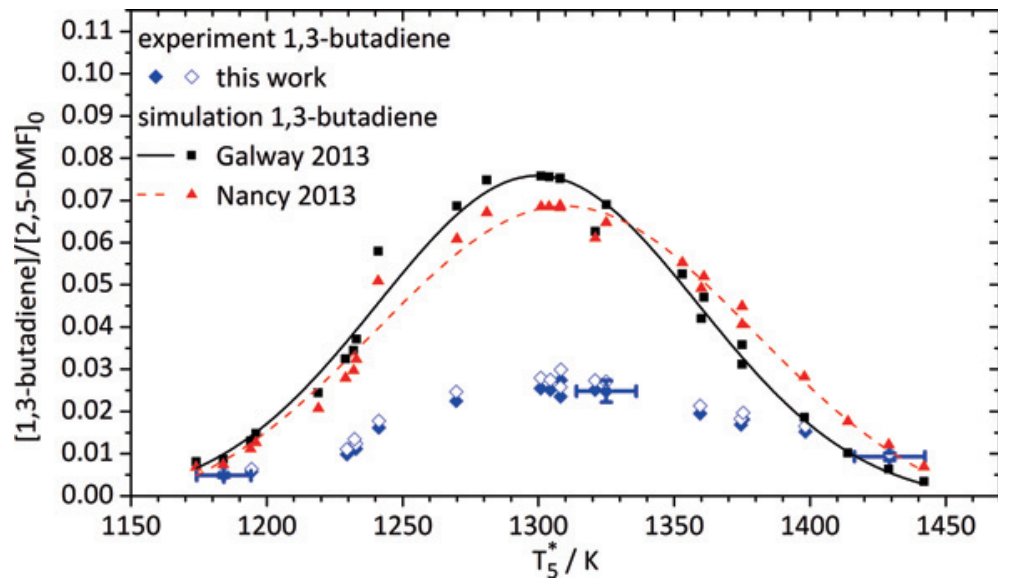

Figure 14: Pyrolysis product 1,3-butadiene: measured (full diamonds) and corrected for dead volume effects (open diamonds). Simulations: Galway 2013 (squares) [6] and Nancy 2013 (triangles) [4].

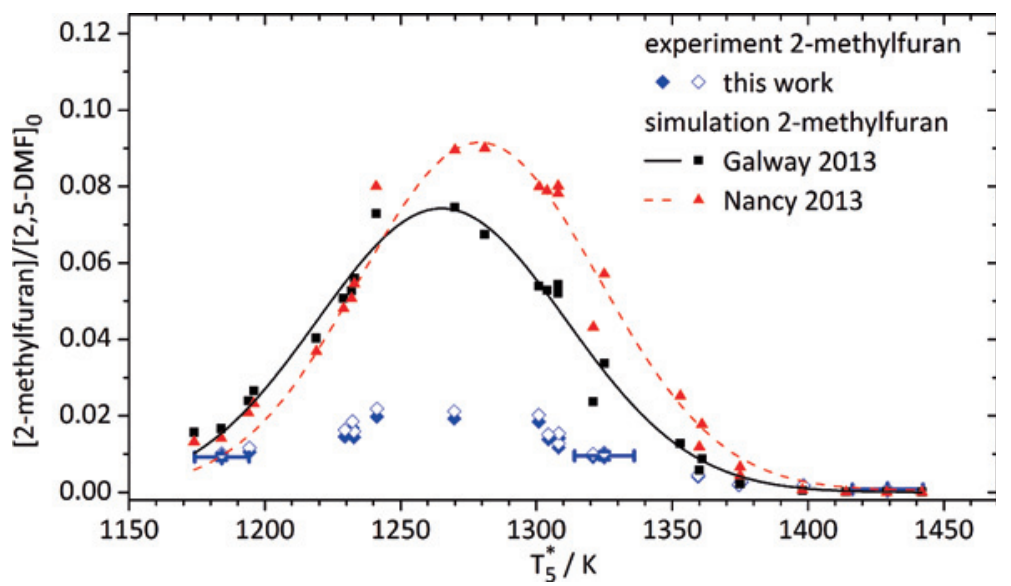

Figure 15: Pyrolysis product 2-methylfuran: measured (full diamonds) and corrected for dead volume effects (open diamonds). Simulations: Galway 2013 (squares) [6] and Nancy 2013 (triangles) [4].

formation at lower temperatures as well as Nancy 2013, but overestimates concentrations at high temperature including the peak value. This tendency can also be observed for 1,3-cyclopentadiene (Figure 10). The position of the maximum is in good agreement with Nancy 2013, the concentration is slightly overpredicted. Galway 2013 matches the slope well up to a temperature of $1300 \mathrm{~K}$ but the maxi- 


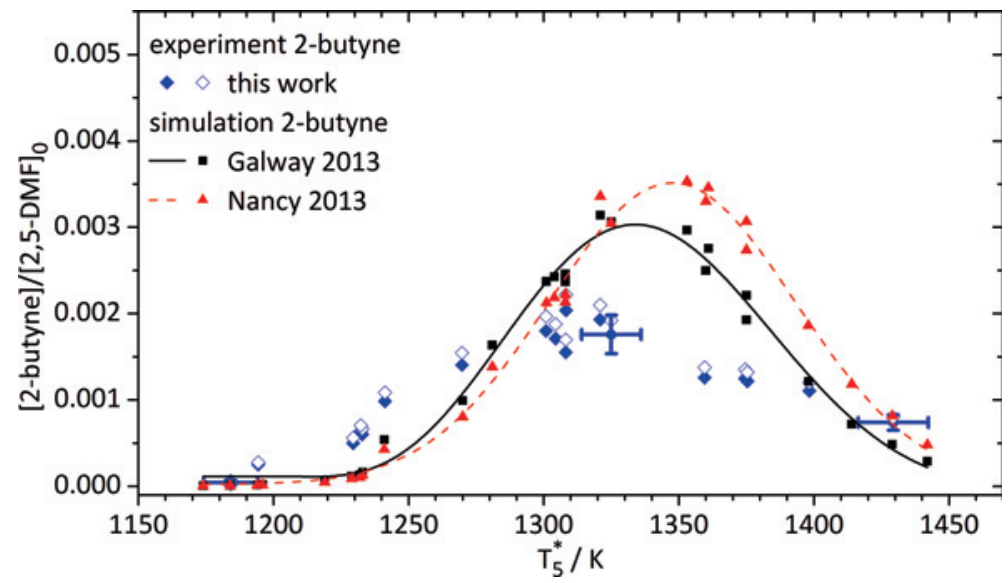

Figure 16: Pyrolysis product 2-butyne: measured (full diamonds) and corrected for dead volume effects (open diamonds). Simulations: Galway 2013 (squares) [6] and Nancy 2013 (triangles) [4].

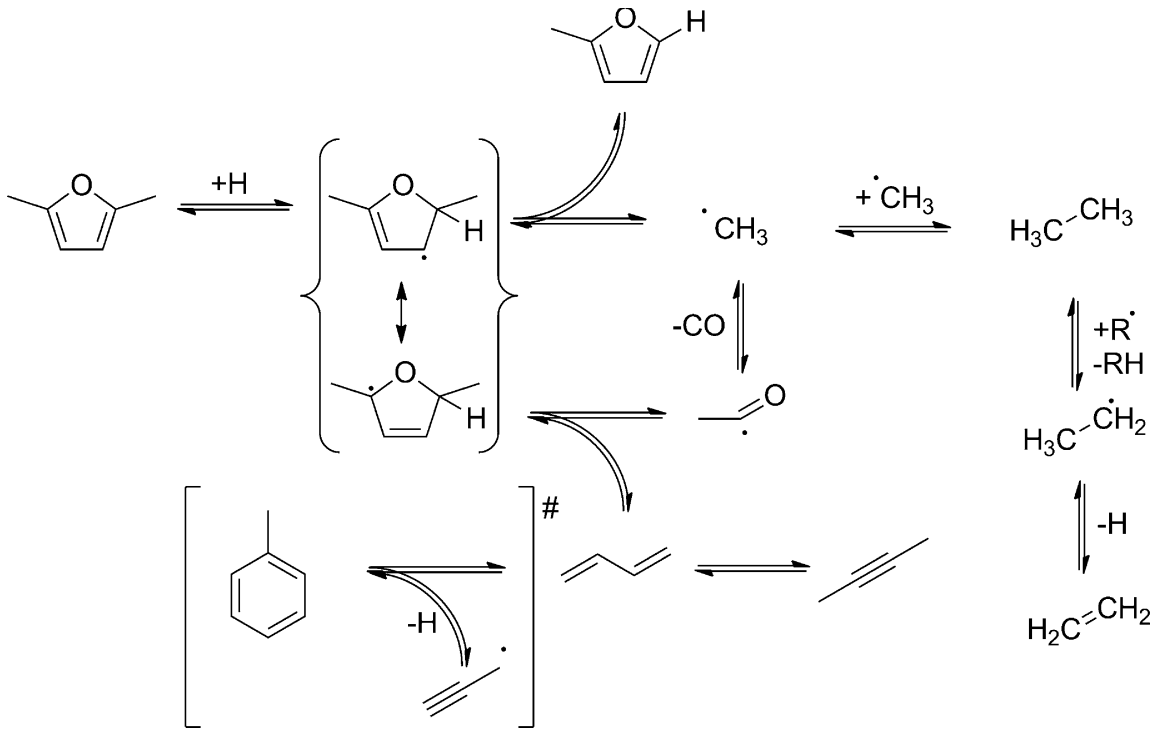

Figure 17: Pathways leading to 1,3-butadiene, 2-methylfuran and 2-methylfuran among other species initiated by hydrogen atom addition to 2,5-DMF. The reaction path labelled \# is only included in Galway 2013. 


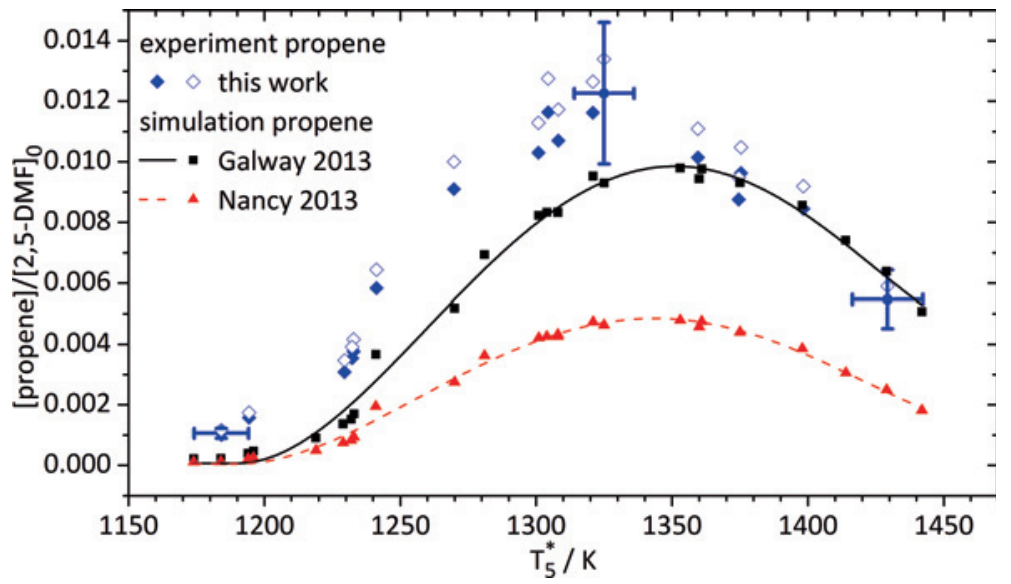

Figure 18: Pyrolysis product propene: measured (full diamonds) and corrected for dead volume effects (open diamonds). Simulations: Galway 2013 (squares) [6] and Nancy 2013 (triangles) [4].

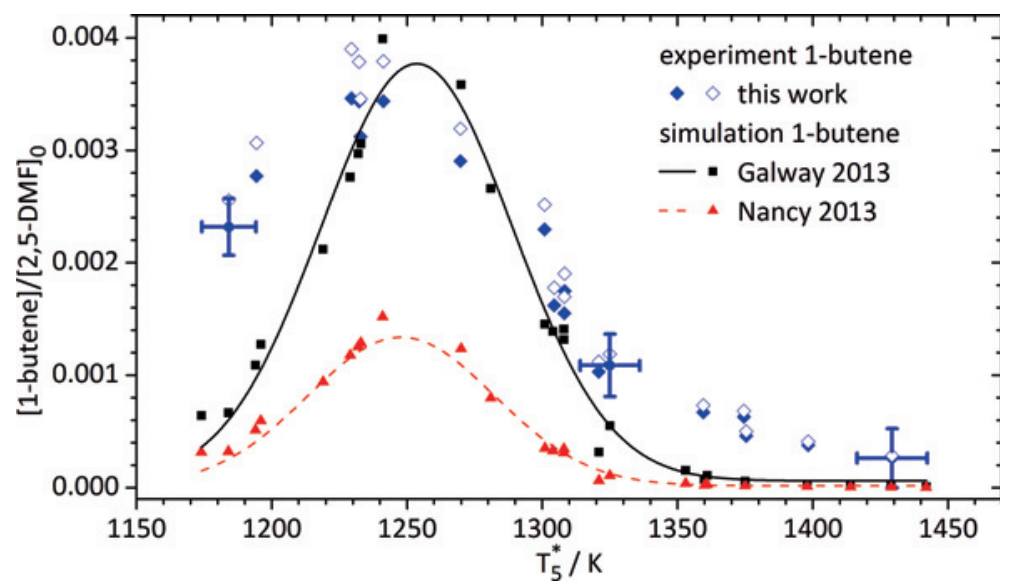

Figure 19: Pyrolysis product 1-butene: measured (full diamonds) and corrected for dead volume effects (open diamonds). Simulations: Galway 2013 (squares) [6] and Nancy 2013 (triangles) [4].

mum is shifted towards higher temperatures. At high temperature, about $1450 \mathrm{~K}$, the main production pathway of 1,3-cyclopentadiene in Galway 2013 is the decomposition route via phenol under scission of carbon monoxide which was studied theoretically by Xu et al. [18]. In the Nancy 2013 mechanism, phenol leads mainly to the phenoxy radical which can isomerize to a bicyclic compound. This pathway leads to 1,3-cyclopentadiene, under carbon monoxide scission, but its contribution is of minor importance, according to reaction rate analysis. The major reac- 


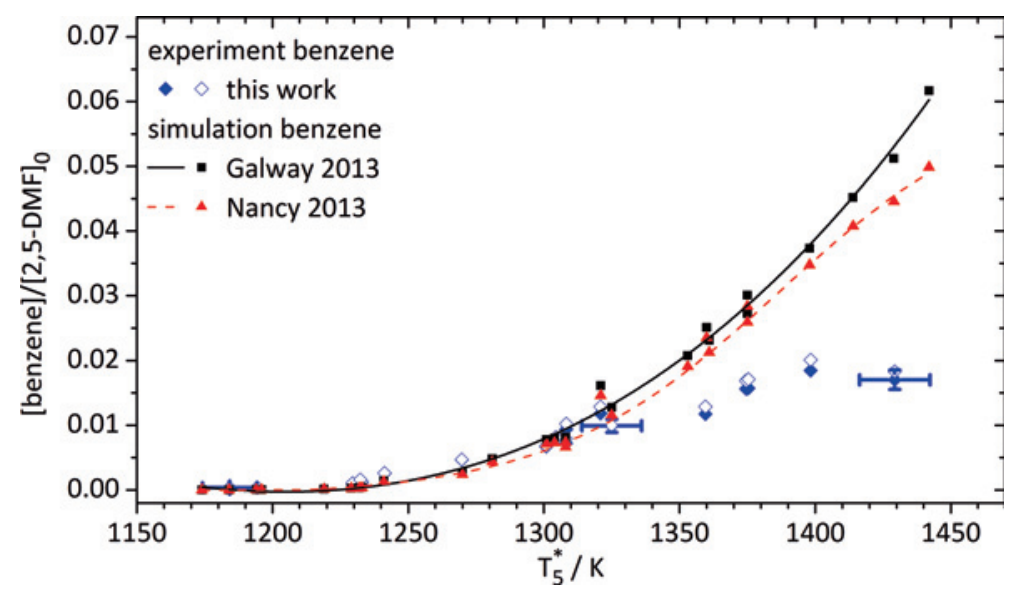

Figure 20: Pyrolysis product benzene: measured (full diamonds) and corrected for dead volume effects (open diamonds). Simulations: Galway 2013 (squares) [6] and Nancy 2013 (triangles) [4].

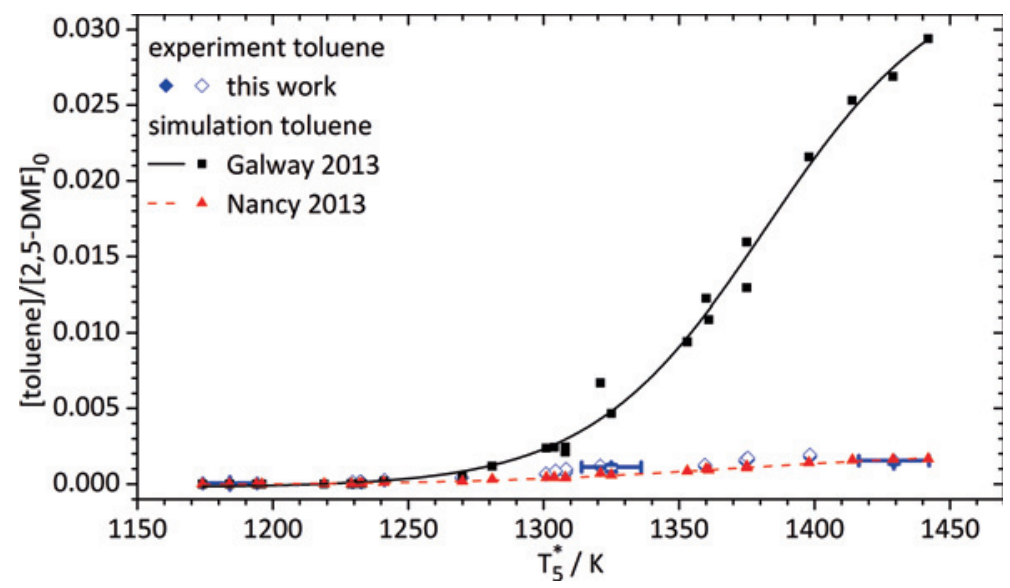

Figure 21: Pyrolysis product toluene: measured (full diamonds) and corrected for dead volume effects (open diamonds). Simulations: Galway 2013 (squares) [6] and Nancy 2013 (triangles) [4].

tants for the production of 1,3-cyclopentadiene in Nancy 2013 are cyclopentenyl isomers. In terms of consumption of 1,3-cyclopentadiene, hydrogen abstraction reactions leading to the 1,3-cyclopentadienyl radical are dominating. Summarizing, the Nancy 2013 implementation of 1,3-cyclopentadiene kinetics seems to be valid in the context of 2,5-DMF-pyrolysis. 
The graph of 1,3-butadiyne (Figure 11) shows good agreement of the results of the experiments with those of the simulations. Above $1400 \mathrm{~K}$, both reaction models tend to overestimate the concentrations measured.

Although Nancy 2013 predicts the maximum concentration of the minor products of the 2,5-DMF pyrolysis, propyne and allene, around $1400 \mathrm{~K}$ in accordance with experiments (Figures 12 and 13), it underestimates the total yields. Galway 2013, on the other hand, is somewhat closer to the absolute yield, but does not capture the profile.

The production of 1,3-butadiene (Figure 14), 2-methylfuran (Figure 15) and 2-butyne (Figure 16), is overestimated by both reaction mechanisms at their maximum concentration. However, both mechanisms reproduce the temperatures of the experimental maximum yields fairly well. The pathways leading to these species are closely linked to each other, as shown in Figure 17, indicating the need for some adjustments to the branching ratios.

For the alkenes propene (Figure 18) and 1-butene (Figure 19), experimental values are much better matched by Galway 2013. Although Nancy 2013 describes well the position of the maximum, it underestimates the product yield over the whole temperature range.

Benzene, an important soot precursor, is well predicted at lower temperatures by both mechanisms, as can be seen in Figure 20. For temperatures above $1350 \mathrm{~K}$, the differences between simulations and experiments get larger.

Toluene (Figure 21) is only a minor component in the pyrolysis of 2,5-DMF. Nevertheless, because of its very high sensitivity in GC/MS analysis, such low concentrations are quantified exactly and revealed some remarkable differences: The Nancy 2013 mechanism is in good agreement with the experimental results. According to our reaction path analysis, most of the toluene is formed from phenol via:

$$
\mathrm{C}_{6} \mathrm{H}_{5} \mathrm{OH}+\mathrm{CH}_{3} \rightarrow \mathrm{C}_{6} \mathrm{H}_{5} \mathrm{CH}_{3}+\mathrm{OH} \text { [19]. }
$$

In the Galway 2013 mechanism, this pathway is not included. The most important pathway there proceeds via the reaction of 1,3-butadiene and the propargyl radical according to

$$
\mathrm{C}_{4} \mathrm{H}_{6}+\mathrm{C}_{3} \mathrm{H}_{3} \rightarrow \mathrm{C}_{6} \mathrm{H}_{5} \mathrm{CH}_{3}+\mathrm{H}[20]
$$

leading to an overestimation of the toluene production. This pathway, currently discussed as a possible important pathway to precursors of soot [21], is not included in the Nancy 2013 model. 


\section{Conclusions}

The pyrolysis of diluted mixtures of 2,5-DMF was studied in the present work by a combination of single pulse experiment and modeling, at temperatures between $1175 \mathrm{~K}$ and $1450 \mathrm{~K}$ and pressures around 8 bar. Sixteen product species were identified and quantified by GC/MS analysis. With respect to the main products, the two literature reaction mechanisms Nancy 2013 [4] and Galway 2013 [6] are in good agreement with the experimental results. For some species, such as ethene, ethyne, and propene, the Galway 2013 mechanism shows a better performance.

At lower temperatures, the Galway 2013 mechanism matches the species profiles of but-1-en-3-yne, 1,3-cyclopentadiene, propyne, and allene but fails to predict their maximum yields correctly, whereas the predictions by the Nancy 2013 mechanism shows a better agreement with respect to the position of the maximum.

For both reaction models, the concentration of the products derived from hydrogen atom addition to 2,5-DMF - 1,3-butadiene, 2-methylfuran, and 2-butyne are overpredicted at the maximum value.

For several minor products, e.g. toluene and 1,3-cyclopentadiene, the simulations based on the mechanisms Nancy 2013 and Galway 2013 show significant deviations from the experimental findings of the present work.

Acknowledgement: Financial support by the Deutsche Forschungsgemeinschaft within the Collaborative Research Centre 606 "Non-Stationary Combustion: Transport Phenomena, Chemical Reactions, Technical Systems" is gratefully acknowledged. The authors thank M. Kapernaum and N. Ackermann for synthesis and handling of the 1,3-butadiyne.

\section{References}

1. Román-Leshkov, Y., Barrett, C. J., Liu, Z. Y., and Dumesic, J. A., Production of dimethylfuran for liquid fuels from biomass-derived carbohydrates, Nature Lett. 447 (2007) 982.

2. Yanowitz, J., Christensen, E., and McCormick, R. L.: Utilization of Renewable Oxygenates as Gasoline Blending Components, National Renewable Energy Laboratory, Technical Report, NREL/TP-5400-50791, (2011).

3. Friese, P., Simmie, J. M., and Olzmann, M.: The reaction of 2,5-dimethylfuran with hydrogen atoms - an experimental and theoretical study, Proceedings of the Combustion Institute 34 (2013) 233.

4. Sirjean, B., Fournet, R., Glaude, P.-A., Battin-Leclerc, F., Wang, W., and Oehlschlaeger, M. A., Shock tube and chemical kinetic modeling study of the oxidation of 2,5-dimethylfuran, J. Phys. Chem. A 117 (2013) 1371. 
5. Lifshitz, A., Tamburu, C., and Shashua, R., Thermal decomposition of 2,5-dimethylfuran. Experimental results and computer modeling, J. Phys. Chem. A 102 (1998) 10655.

6. Somers, K. P., Simmie, J. M., Gillespie, F., Conroy, C., Black, G., Metcalfe, W. K., BattinLeclerc, F., Dirrenberger, P., Herbinet, O., Glaude, P.-A., Dagaut, P., Togbe, C., Yasunaga, K., Fernandes, R. X., Lee, C., Tripathi, R., and Curran, H. J., A comprehensive experimental and detailed chemical kinetic modelling study of 2,5-dimethylfuran pyrolysis and oxidation, Combustion and Flame 160 (2013) 2291.

7. Somers, K. P., Simmie, J. M., Metcalfe, W. K., and Curran, H. J., The pyrolysis of 2-methylfuran: a quantum chemical, statistical rate theory and kinetic modelling study, Phys. Chem. Chem. Phys. 16 (2014) 5349.

8. Schuler, D. F., PhD thesis, Universität Stuttgart, Germany, forthcoming 2015.

9. Frank, P. and Just, T., High-temperature reaction-rate for $\mathrm{H}+\mathrm{O}_{2}=\mathrm{OH}+\mathrm{O}$ and $\mathrm{OH}+\mathrm{H}_{2}=$ $\mathrm{H}_{2} \mathrm{O}+\mathrm{H}$, Ber. Bunsenges. Phys. Chem. 89 (1985) 181.

10. Lifshitz, A. (Ed.): Shock Waves in Chemistry, Marcel Dekker, Inc., New York (1981).

11. Johnson, A. W., 218. 2-butyne-1: 4-diol. Part I. Reactions of the hydroxyl groups, The Royal Society of Chemistry, J. Chem. Soc. (1946) 1009.

12. Verkruijsse, H. D. and Brandsma, L., A detailed procedure for the preparation of butadiyne, Synthetic Communications 21 (1991) 657.

13. Tsang, W. and Herron, J. T., Chemical kinetic data base for propellant combustion. I. Reactions involving $\mathrm{NO}, \mathrm{NO}_{2}, \mathrm{HNO}, \mathrm{HNO}_{2}, \mathrm{HCN}$ and $\mathrm{N}_{2} \mathrm{O}$, J. Phys. Chem. Ref. Data 20 (1991) 609.

14. Dagaut, P., Karsenty, F., Dayma, G., Diévart, P., Hadj-Ali, K., Mzé-Ahmed, A., BraunUnkhoff, M., Herzler, J., Kathrotia, T., Kick, T., Naumann, C., Riedel, U., and Thomas, L., Experimental and detailed kinetic model for the oxidation of a gas to liquid (GtL) jet fuel, Combustion and Flame 161 (2014) 835.

15. Lutz, A. E., Kee, R. J., and Miller, J. A., SENKIN: a Fortran Program for Predicting Homogeneous Gas Phase Chemical Kinetics with Sensitivity Analysis, Report 87-8248, Sandia National Laboratories, Livermore (1988).

16. Deminsky, M., Chorkov, V., Belov, G., Cheshigin, I., Knizhnik, A., Shulakova, E., Shulakov, M., Iskandarova, I., Alexandrov, V., Petrusev, A., Kirillov, I., Strelkova, M., Umanski, S., and Potapkin, B., Comput. Mater. Sci. 1692003 Chemical WorkBench 4.0, Kintech Laboratory, http://www.kintechlab.com/products/chemical-workbench.

17. Hidaka, Y., Nakamura, T., Miyauchi, A., Shiraishi, T., and Kawano, H., Thermal decomposition of propyne and allene in shock waves. I., J. Chem. Kinet. 21 (1989) 643.

18. Xu, Z. F. and Lin, M. C., Ab initio kinetics for the unimolecular reaction $\mathrm{C}_{6} \mathrm{H}_{5} \mathrm{OH} \rightarrow \mathrm{CO}+$ $\mathrm{C}_{5} \mathrm{H}_{6}$. J. Phys. Chem. A 110 (2006) 1672.

19. Tian, Z., Pitz, W. J., Fournet, R., Glaude P.-A., and Battin-Leclerc, F., A detailed kinetic modeling study of toluene oxidation in a premixed laminar flame, Proceedings of the Combustion Institute 33 (2011) 233.

20. Laskin, A., Wang, H., and Law, C. K., Detailed kinetic modeling of 1,3-butadiene oxidation at high temperatures, Int. J. Chem. Kinet. 32 (2000) 589.

21. Hansen, N., Braun-Unkhoff, M., Kathrotia, T., Lucassen, A., and Yang, B., Understanding the reaction pathways in premixed flames fueled by blends of 1,3-butadiene and n-butanol, Proc. Comb. Inst. 35 (2014), online available: DOI: 10.1016/j.proci.2014.05.005. 